\title{
Produção de Jogos Digitais Educativos por Alunos do Ensino Superior: Um Relato da Experiência
}

\author{
Kleber Tavares Fernandes ${ }^{1,2}$, Eduardo Henrique da Silva Aranha ${ }^{1}$, \\ Márcia Jacyntha Nunes Rodrigues Lucena ${ }^{1}$ \\ ${ }^{1}$ Programa de Pós-Graduação em Sistemas e Computação - PPgSC \\ Universidade Federal do Rio Grande do Norte - UFRN \\ Caixa Postal 1524 -Lagoa Nova - CEP 59072-970 - Natal/RN - Brasil \\ ${ }^{2}$ Departamento de Ciências Exatas e Tecnologia da Informação \\ Universidade Federal Rural do Semiárido - UFERSA \\ CEP 59515-000 - Campus Angicos/RN - Brasil
}

Kleber76@gmail.com, eduardoaranha@dimap.ufrn.br, marciajedimap.ufrn.br

\begin{abstract}
The development of a game is a complex activity that involves several phases and professionals from different areas. Some computer science courses have benefited by using digital game development practices associated with theories presented in the classroom environment. In this context, this article reports an experience of the practical training of computing students in a university using as a teaching-learning strategy the development of digital games directed for educational.
\end{abstract}

Resumo. O desenvolvimento de um jogo é uma atividade complexa que envolve diversas etapas e profissionais de diferentes áreas. Algumas disciplinas dos cursos de computação têm se beneficiado quando adotam práticas de desenvolvimento de jogos digitais associadas às teorias apresentadas em sala de aula. Neste contexto, este artigo relata uma experiência da formação prática de alunos da área de computação de uma Universidade Brasileira utilizando como estratégia de ensino aprendizagem a produção de jogos digitais com fins educacionais.

\section{Introdução}

A maioria dos projetos pedagógicos dos cursos de computação visa a formação de profissionais que sejam capazes de analisar problemas diversos e propor soluções computacionais eficientes, rápidas e viáveis economicamente. Estes projetos valorizam a participação dos alunos em experiências práticas associadas aos conteúdos das disciplinas e contribuindo para uma formação mais próxima do que a indústria exige.

Nesse sentido as disciplinas de programação, engenharia de software, entre outras, têm buscado relacionar os conteúdos teóricos, vistos em sala de aula, com momentos práticos nos laboratórios de informática, criando oportunidade para os alunos experimentarem a prática no desenvolvimento de sistemas computacionais. Contudo, a formação oferecida constitui-se insuficiente em função da reduzida carga horária 
destinada às experiências práticas e a falta de diálogo entre a academia e a indústria [Begosso et al 2011].

Diante disso, algumas pesquisas tem buscado propor momentos extracurriculares para que os alunos possam vivenciar a prática em desenvolvimento de sistemas computacionais. Esses momentos têm despertado nos alunos uma curiosidade e motivação pela área constituindo-se, muitas vezes, como um diferencial no seu desempenho e na sua formação profissional [Paes et al 2013], [Torezani et al 201] e [Nascimento et al 2010].

Neste contexto, disciplinas de programação têm se beneficiado pelo interesse dos alunos por jogos digitais. Eles se sentem cada vez mais atraídos por disciplinas com teor prático que utilizam o desenvolvimento de jogos como recurso para apresentar os conteúdos [Prensky 2012]. Apesar desta atratividade por parte dos alunos, o desenvolvimento de jogos em disciplinas práticas de computação não é uma atividade simples, pois envolve diversas etapas e profissionais de várias áreas, trazendo à tona a complexidade que contém projetos reais.

Além disso, a grande variedade dos gêneros e estilos de jogos, como também a definição dos seus requisitos tornam os projetos mais desafiadores, muitas vezes sendo necessário o envolvimento de outras disciplinas, tornando o projeto uma atividade interdisciplinar.

Portanto, segundo Prensky (2012), a estratégia do uso de jogos na formação prática de alunos dos cursos de computação tem-se mostrado como um elemento motivacional, interdisciplinar bastante envolvente. Têm buscado resolver os problemas de formação desses alunos tentando aproximar a indústria da academia, bem como proporcionam oportunidades para realização de atividades interdisciplinares durante $o$ curso, constituindo-se como momentos de significativa aprendizagem.

Este artigo relata uma experiência da formação prática de alunos de cursos superiores da área de computação de uma Universidade Brasileira, utilizando como estratégia de ensino-aprendizagem a produção de jogos digitais com fins educacionais. Está organizado da seguinte maneira: a seção 2 descreve a fundamentação teórica utilizada neste trabalho; a seção 3 apresenta a estratégia de ensino aprendizagem através do desenvolvimento de jogos; a seção 4 relata a experiência da aplicação da estratégia com um grupo de universitários, bem como os resultados alcançados; e, finalmente, a seção 5 apresenta as considerações finais e trabalhos futuros.

\section{Fundamentação Teórica}

O Brasil tem-se destacado no mercado Internacional como um dos produtores de software em potencial em razão da disponibilidade de mão de obra e da produção a baixo custo. Porém, a formação insuficiente de profissionais qualificados na área de TIC, como programadores, pode-se caracterizar como fator de entrave no desenvolvimento do País [Vivaqua, 2009].

Para evitar que isso aconteça, os cursos de computação das Universidades Brasileiras têm desempenhado um importante papel na formação de profissionais qualificados para atuar na indústria de softwares.

Preparar os alunos desses cursos para o mercado de trabalho é um desafio. A falta de diálogo entre a academia e a indústria, como também a falta de recursos das 
Universidades, têm dificultado a oferta de atividades que criam oportunidades de formação mais próximas do perfil exigido pela indústria de software. Além disso, a carga horária das disciplinas de caráter prático dos cursos de computação parece ser insuficiente comprometendo a formação dos alunos. Segundo Begosso et al (2011), isso faz com que os alunos concluam seus cursos sem a maturidade que a indústria exige.

A saída adotada por algumas Instituições de Ensino Superior para minimizar esse problema tem sido a oferta de momentos de formação prática em desenvolvimento de softwares.

O trabalho de Barth et al (2011) apresenta um programa de formação ofertado semestralmente aos alunos do curso de Análise e Desenvolvimento de Software. Nesse programa, os alunos participam do desenvolvimento de um software sob demanda. Como resultado é perceptível a melhoria no desempenho dos alunos nas disciplinas, a formação de um portfólio pessoal, a prática em linguagens de programação e melhor compreensão sobre requisitos.

A pesquisa de Begosso et al (2011) descreve o projeto de implantação do Programa de Residência em Software em um ambiente de graduação, permitindo aos estudantes a participação no desenvolvimento de projetos de software para empresas reais. O trabalho constatou um aumento da maturidade dos alunos e a aproximação da academia com o mercado de trabalho.

Já Way (2005) relata uma experiência de alunos do curso de Engenharia de Software na participação de um programa de desenvolvimento de softwares. $O$ programa proporcionou a vivência no desenvolvimento de um projeto de sistemas.

O trabalho de Sampaio et al (2005) apresenta um programa de residência de software denominado Programa de Teste de Software. Os alunos eram treinados nas diversas áreas do desenvolvimento de sistemas, porém o foco principal era a disciplina de teste de software.

A diferença da experiência relatada neste artigo para as iniciativas apresentadas acima está na estratégia da produção de jogos digitais como motivação para a prática de desenvolvimento de softwares. Também a constituição de uma equipe multidisciplinar formada por alunos e profissionais que atuaram em diversos papéis, assim como realizado na indústria de jogos.

Atualmente, pesquisas demonstram uma série de benefícios da inclusão do desenvolvimento de jogos digitais no processo ensino-aprendizagem de computação. Apontam possibilidades de tornarem-se um rico instrumento para a construção do conhecimento em diversas disciplinas. Fazer seu próprio jogo possibilita ao aluno alguns benefícios: podem ser ferramentas eficientes, pois eles divertem enquanto programam e testam os jogos, facilitam a aprendizagem e aumentam a capacidade de retenção do que é ensinado, exercitando as funções mentais e intelectuais do aluno [Sá et al 2007] e [Tarouco et al 2004].

Além disso, com o aumento da demanda por jogos cada vez mais sofisticados e a possibilidade de usá-los na educação surge a necessidade do envolvimento de outros profissionais, tais como educadores, designers, editores de áudio, entre outros, para tornar os jogos mais atrativos, divertidos e coerentes com as questões pedagógicas. $\mathrm{O}$ envolvimento dos alunos de computação com esses profissionais constitui-se como momentos de interação bastante ricos e com enorme potencial de aprendizado. 


\section{Estratégia de Ensino-Aprendizagem Através da Produção de Jogos}

Os conteúdos vistos nas disciplinas dos cursos de computação de grande parte das instituições de ensino superior não têm acompanhado a evolução que essa área tem passado, nem tão pouco as demandas e os conhecimentos exigidos pela indústria. Uma das estratégias de aproximar a indústria e a academia é a oferta de programas de treinamento em desenvolvimento de software. Tais programas têm o objetivo principal de possibilitar aos alunos a capacitação nas tecnologias utilizadas pela indústria de forma prática. Os alunos participantes do programa, orientados pelos professores, vivenciaram experiências práticas em projetos de desenvolvimento de softwares, em todas as suas fases, simulando na academia um ambiente da indústria. Dessa maneira, o programa contribui para a formação e inserção desses alunos no mercado de trabalho.

Nesse sentido, a estratégia adotada pela Universidade, onde foi realizada a experiência relatada neste trabalho, foi a oferta de um programa de treinamento em desenvolvimento de jogos. Inicialmente os alunos foram selecionados para compor equipes de desenvolvimento que, sob orientação de um professor, vivenciaram experiências práticas na produção de jogos demandados pela indústria. A primeira etapa do programa foi constituída de um treinamento em desenvolvimento de jogos que contempla ferramentas, metodologias, análise e gestão de projetos. A segunda fase foi o desenvolvimento de um jogo educativo. Cada equipe teve a oportunidade de participar do programa que durou aproximadamente um semestre, podendo se estender aos semestres seguintes, até a conclusão do curso.

A equipe de produção foi constituída por alunos dos cursos de computação que foram divididos de acordo com os seguintes perfis: designers gráficos, programadores, sonoplastas, game designers e gerente de projeto. Os perfis e respectivas responsabilidades são descritos a seguir. O programador é responsável pela codificação (programação) dos jogos em uma linguagem de programação específica. Enquanto o designer gráfico é responsável pela concepção e produção da arte gráfica dos jogos, incluindo os cenários, personagens e demais elementos gráficos do jogo. Já o sonoplasta é responsável pela concepção e produção da trilha sonora dos jogos, incluindo as músicas e os sons vinculados aos elementos dos jogos. O game designer é responsável pelo projeto de criação do jogo, inclui a definição da história, roteiro, regras, animação dos personagens, planejamento da interface, além de garantir a interatividade e entretenimento do jogo. $\mathrm{O}$ gerente do projeto é responsável pelo gerenciamento do projeto e acompanhamento das fases do desenvolvimento dos jogos e finalmente o testador e/ou os avaliadores são responsáveis pelos testes e validação dos jogos e pode ser um pedagogo, coordenador, game designer, programador, professor e/ou aluno.

Os professores envolvidos no programa identificaram as expectativas e necessidades de desenvolvimento integral dos alunos e articularam oportunidades educativas capazes de atendê-las. Acompanharam e orientaram o desenvolvimento dos jogos. Articularam o conteúdo teórico das disciplinas com a prática da indústria.

O projeto contou também com uma pedagoga responsável pelo levantamento dos requisitos pedagógicos do jogo. Incluiu a análise do material didático, fonte de inspiração do jogo, inicialização e acompanhamento do cartão do jogo (considerado o documento de requisitos para o projeto) e a avaliação do produto final para prováveis adaptações ou melhorias. 
O ambiente de desenvolvimento dos jogos foi formado pelas seguintes ferramentas: ${ }^{1}$ Construct 2 , que permite a criação dos jogos em 2 dimensões no formato HTML5; ${ }^{2}$ Ilustrator, utilizado para produção dos elementos gráficos do jogo; ${ }^{3}$ Audacity, utilizado na produção dos áudios; e ${ }^{4}$ Trello, que permite o gerenciamento das tarefas e do fluxo de produção. Estas ferramentas foram escolhidas pela facilidade de uso e vasto material didático disponível na literatura e internet.

O documento de requisitos para a elaboração dos jogos denomina-se nesta experiência de "Cartão do Jogo". Nele contém a identificação do material didático utilizado como ponto de partida, público alvo, objetivos, conteúdos e habilidades a serem trabalhadas, as variações de dificuldade, formas de avaliação da aprendizagem, a proposta do jogo, itens do game designer, projeto gráfico, projeto do áudio, requisitos de programação e arquitetura, regras e créditos do jogo.

\subsection{Visão Geral do Processo de Desenvolvimento}

Para a produção de jogos é necessário utilizar um processo de desenvolvimento de software que se adapte à realidade do projeto. O processo utilizado no programa de treinamento foi elaborado especificamente para essa finalidade através da colaboração da equipe de alunos e professores envolvidos. Foram realizadas diversas reuniões usando a técnica de brainstorming para definição das fases e atividades do processo, bem como a atuação da equipe. A Figura 1 apresenta o processo de desenvolvimento de jogos usado nesta experiência de aprendizado.

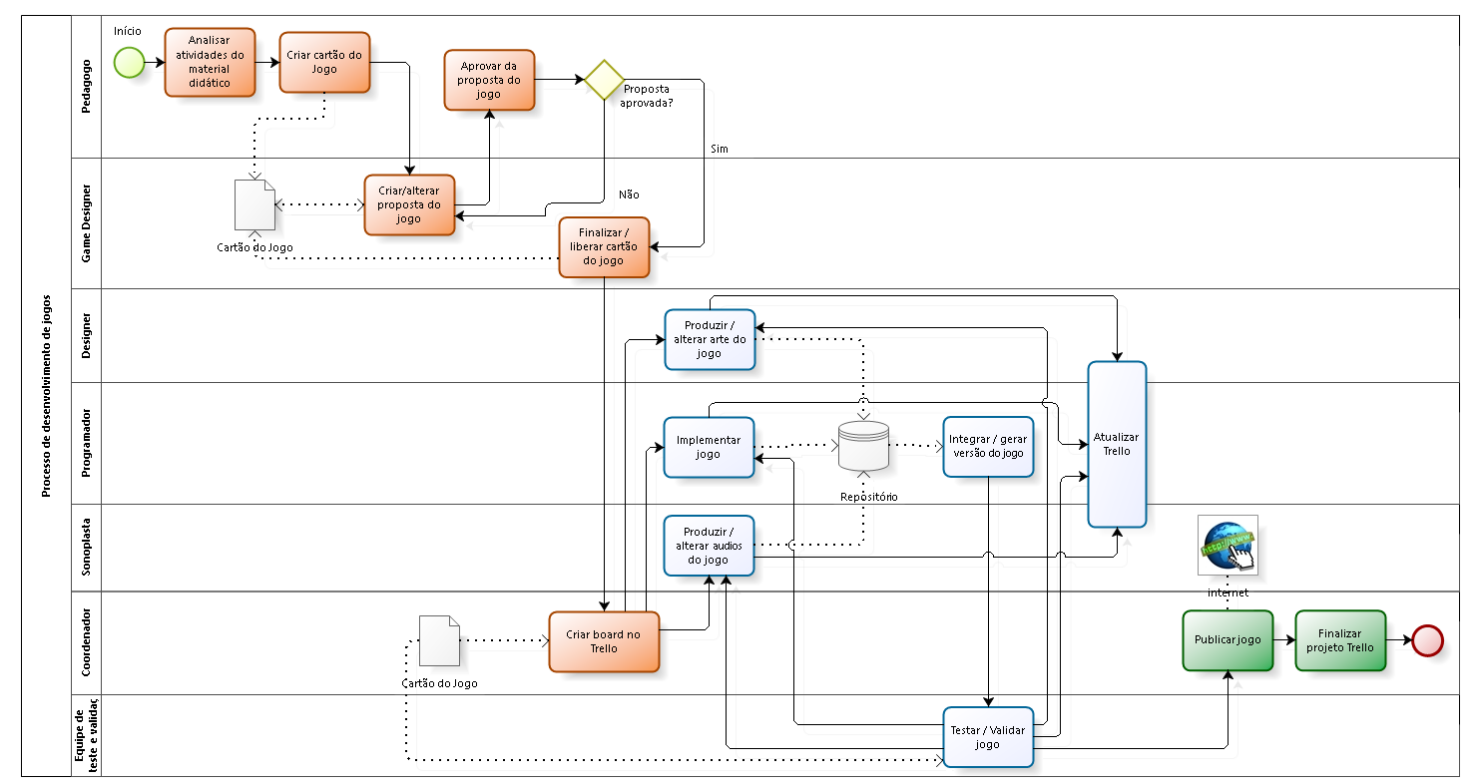

Figura 1. Processo de Desenvolvimento de Jogos

A Figura 1 apresenta as fases e atividades divididas em raias que representam os perfis dos membros da equipe envolvida no projeto. As atividades relacionadas à préprodução estão na cor laranja, as de produção estão na cor azul e as da pós-produção

\footnotetext{
${ }^{1} \mathrm{https}: / / \mathrm{www} \cdot$ scirra.com/construct2

${ }^{2} \mathrm{https}: / /$ www.adobe.com/

${ }^{3} \mathrm{http}: / /$ www.audacityteam.org/

${ }^{4}$ https://trello.com/
} 
estão na cor verde. Algumas atividades não são necessariamente sequenciais para dar maior agilidade ao desenvolvimento do jogo.

O processo inicia na fase de pré-produção com a análise das atividades do material didático pela pedagoga. Essas atividades representam a demanda inicial, ou o que se espera que o aluno (jogador) aprenda em determinado ano ou série. Elas norteiam ou inspiraram a definição dos objetivos, habilidades ou conteúdos a serem abordados nos jogos. Vários livros didáticos da mesma série foram consultados, para ampliação das referências.

Após a análise, a pedagoga cria um cartão com os requisitos iniciais do jogo. A partir desse cartão é possível iniciar as atividades do game designer que cria a proposta de desenvolvimento do jogo. A proposta complementa do cartão do jogo é então analisada podendo ser aprovada ou não pela pedagoga, de acordo com o atendimento dos requisitos levantados. Sendo aprovada segue para finalização e liberação do cartão estando pronto para iniciar a fase de produção do jogo. Caso contrário, retorna para o game designer realizar as alterações necessárias e ser novamente avaliado.

A fase de produção é iniciada com 3 processos executados em paralelo que são: produção da arte gráfica do jogo, produção dos áudios do jogo e a implementação do jogo. A produção da arte gráfica consiste em elaborar todos os elementos gráficos do jogo. A produção dos áudios consiste na elaboração da trilha sonora e todos os sons que irão compor o jogo. A tarefa de implementar o jogo consiste na sua codificação em uma linguagem de programação.

Os produtos finais dessas 3 atividades são armazenados em um repositório do projeto. Assim, é possível integrar os elementos gráficos e sons ao código fonte e gerar a primeira versão executável do jogo.

Ainda na fase de produção são realizados os testes e validação do jogo. A partir dos testes são verificados problemas na mecânica do jogo, erros de programação, problemas de design gráfico e no áudio do jogo. Outra verificação feita é se os requisitos descritos no cartão do jogo foram atendidos. Não havendo nenhum problema detectado pelos testes o jogo é validado. Caso contrário, é encaminhado para a realização das correções necessárias. O registro dos erros é realizado no Trello. Essa tarefa é realizada por uma equipe constituída pela pedagoga, gerente do projeto, game designer, programador, professores e alunos.

À medida que o jogo vai sendo desenvolvido é possível atualizar as tarefas que foram cadastradas no Trello. A pedagoga também interage com a equipe fornecendo orientações no decorrer do processo. Dessa forma, o gerente do projeto tem condições de acompanhar a produtividade da equipe, os problemas relatados e suas soluções. A fase final do processo é a pós-produção que possui como tarefas principais a publicação do jogo numa plataforma computacional ou site na Internet e a finalização do projeto no Trello.

\section{Relato da Experiência da Produção de Jogos}

O ponto de partida para iniciar este trabalho foi o desenvolvimento de jogos digitais educativos a partir do material didático do componente curricular de matemática, das séries iniciais do ensino fundamental, por alunos do Ensino Superior. Tivemos como demandante uma escola pública da rede municipal de ensino que reuniu sua equipe de 
professores e coordenadores para definir os requisitos necessários para elaboração dos jogos.

O componente curricular da matemática foi escolhido em razão do baixo rendimento dos alunos nas avaliações realizadas pela escola. Ela espera que a aplicação dos jogos desenvolvidos nas aulas de matemática possa contribuir para a aprendizagem dos alunos e melhoria dos índices avaliativos.

Os jogos produzidos seguiram o processo de desenvolvimento de jogos educativos proposto pelo programa de treinamento. Dessa forma, pretendeu-se garantir uma produção padronizada, em curto prazo de tempo e que pudesse atender efetivamente aos requisitos do projeto.

A equipe foi formada por 17 alunos do curso superior de computação de uma Universidade Brasileira sendo dividida em 07 grupos de produção. Cada grupo foi constituído por alunos com o perfil de desenvolvedor, design gráfico, game designer e sonoplasta. O programa contou com um profissional da pedagogia que prestava $o$ suporte pedagógico aos grupos. Todos eram acompanhados por um aluno que fazia o papel de gerente de projetos.

A produção dos jogos foi realizada em ciclos produtivos. Cada um deles com duração média de 4 semanas de trabalho. Cada grupo era responsável por desenvolver 1 jogo por ciclo, cumprindo todas as etapas de produção já descritas. A experiência foi realizada num período de 3 ciclos produtivos. A equipe e os ciclos produtivos foram organizados e gerenciados pelo gerente de projetos utilizando uma ferramenta de gestão de tarefas (Trello). A cada semana eram realizadas reuniões para monitorar o progresso da produção dos jogos, semelhante as sprints da metodologia ${ }^{5}$ Scrum.

Foram realizadas reuniões avaliativas onde a equipe avaliava a eficiência do processo e a qualidade dos jogos produzidos ao final de cada ciclo realizado. Para iniciar o ciclo seguinte, era necessário fechar o ciclo anterior, fazendo os ajustes ou alterações percebidas nos testes e avaliação dos jogos.

\subsection{Avaliação dos Resultados Alcançados}

Esta subseção apresenta os resultados alcançados, a avaliação dos jogos produzidos e do processo utilizado. Essa atividade foi realizada através da análise dos questionários e entrevistas respondidas pelas equipes participantes do projeto. Ao final de cada ciclo de desenvolvimento foi aplicado um questionário e ao final do projeto foram realizadas entrevistas com os grupos de produção.

\subsubsection{Considerações a Respeito dos Jogos Desenvolvidos}

A partir da análise dos jogos produzidos, é possível afirmar que as equipes estiveram voltadas para o atendimento dos objetivos, conteúdos e habilidades estabelecidos inicialmente no cartão do jogo. Na etapa de concepção do tema a ser abordado, personagens, cenários, procedimentos envolvidos no jogo, título, houve um verdadeiro desdobramento para se idealizar um produto criativo, que atendesse à faixa etária e aos objetivos pretendidos. Verificamos interfaces bem apresentadas, propostas criativas de jogo, com temas e títulos adequados aos interesses dos alunos (jogadores). Portando, observamos que a fase de pré-produção do processo proposto está clara e possui tarefas

\footnotetext{
${ }^{5} \mathrm{http} / /$ www.desenvolvimentoagil.com.br/scrum/
} 
compatíveis para o desenvolvimento de jogos dessa natureza.

Ao verificar o conjunto dos jogos, percebemos a variedade dos conteúdos que foi proposto, o que possibilita a abordagem de diversos assuntos do programa das séries iniciais do ensino fundamental por meio dos jogos.

Porém, como análise do resultado da fase de produção, constatamos que 3 jogos não conseguiram corresponder ao produto idealizado, no que se refere ao propósito educacional de desenvolver habilidades e favorecer a aprendizagem. Apesar do empenho da equipe na elaboração do cartão do jogo, a atividade de implementação, em 4 jogos, foi comprometida pela limitação de conhecimento avançado da ferramenta utilizada e do tempo destinado para essa atividade. Esses jogos requereram ações mais complexas que exigiam mais tempo de implementação.

Outro ponto que merece destaque, em 5 jogos, é a baixa interação entre o jogo e o jogador, sobretudo no início, quando deveria fornecer informações sobre as regras, desafios e os controles do jogo. Percebemos também a necessidade de melhoria no feedback para atender ao propósito de comunicação e para motivar o jogador a continuar jogando.

\subsubsection{Considerações a Respeito do Processo Utilizado}

$\mathrm{O}$ resultado das entrevistas realizadas com a equipe leva a perceber que o processo utilizado nesta experiência favorece a produção completa dos jogos, indicando que a sequência de atividades foi adequada. Aponta-se que o processo permite uma comunicação efetiva entre os seus participantes.

O processo contempla a possibilidade de retorno à atividade anterior para realização de ajustes ainda durante o desenvolvimento do jogo. Isso foi apontado positivamente pelos entrevistados. As atividades produzir/alterar arte do jogo, implementar jogo e produzir/alterar áudios do jogo podem ser executadas em paralelo o que permite agilizar a fase de produção. Porém, a tarefa de integrar/gerar versão do jogo pode ser comprometida por falhas na entrega dos artefatos que a antecede.

Fazendo uma análise do processo por fases, foi possível tecer ainda algumas considerações. Por exemplo, o cumprimento adequado da fase de pré-produção pelos profissionais envolvidos. Todas as atividades foram cumpridas satisfatoriamente. As atividades da produção também foram desenvolvidas de acordo com o previsto, com ressalva para a última atividade - testar e validar jogo; A equipe de teste não foi constituída por todos os membros idealizados. Os jogos produzidos não foram testados por professores e alunos. Este fator influenciou de forma negativa a finalização dos jogos, considerando que tais atores são o público alvo de toda a produção; 11 jogos não retornaram para a validação da pedagoga, a quem caberia uma análise mais cuidadosa quanto ao atendimento dos requisitos e uma intervenção/orientação para aprimoramento. Sem tal análise, não foi possível fazer o aperfeiçoamento mais apurado, que ficou centrado nas percepções das demais pessoas da equipe, as quais não tinham formação pedagógica. As deficiências nos testes comprometeram o êxito da fase de pósprodução, tendo em vista que sem a finalização adequada, os jogos não podiam ser publicados e disponibilizados para o uso. 


\subsubsection{Considerações a Respeito da Estratégia de Formação dos Alunos}

A realização do programa de treinamento em desenvolvimento de jogos constituiu-se como uma experiência formativa para todos os envolvidos: alunos e professores.

Os depoimentos relatados nas entrevistas realizadas com os alunos acerca da sua participação e aprendizagem durante a experiência mostra que foram proporcionadas condições muito aproximadas da realidade com a qual poderão se deparar na indústria. Isso porque, estiveram envolvidos nesse programa: atendimento à demanda de clientes, necessidade de alinhamento de procedimentos adotados, necessidade de clareza e definição dos papéis de todos os membros da equipe, necessidade de tomar decisões em grupo, avaliar os resultados, retomar o processo e validar os jogos. Para os alunos, foi possível aprender na prática os conteúdos envolvidos em cada item do programa.

Para os professores, a estratégia adotada proporcionou uma formação mais consistente aos alunos, por possibilitar a associação entre teoria e prática, além da aquisição de expertise no desenvolvimento de jogos. A atuação em diversos perfis também foi importante, pois possibilitou compartilhar ideias e buscar saberes de áreas distintas em prol de um objetivo em comum: a criação de jogos para uma clientela definida. Tal vivência, certamente colaborou para ampliar a compreensão dos conceitos trabalhados nas disciplinas. Isso ficou evidenciado no resultado final da avaliação da aprendizagem das disciplinas dos alunos envolvidos no programa, em relação aos demais. Por essa razão, entende-se que essa experiência também trouxe contribuições para os professores, que puderam dar sentido às suas aulas e acompanhar, na prática, a atuação dos seus alunos na aplicação dos conceitos aprendidos.

\section{Conclusões}

Diversos autores ressaltam a importância do desenvolvimento de jogos como estratégia de ensino aprendizagem por possibilitar ao aluno a oportunidade de participar da construção do seu conhecimento [Sutherland 2014] [Savi et al 2014]. Porém, nossos estudos apontam a ausência de processos sistemáticos para desenvolver jogos digitais educativos que atendam tanto às necessidades técnicas e pedagógicas. Verifica-se a necessidade de aprofundamento do diálogo entre os profissionais de educação e de tecnologia da informação, para garantir a concepção de produtos que tanto atendam às demandas educacionais, como também promovam a inserção dos alunos no "universo tecnológico".

A experiência aqui relatada constitui-se como modelo de consolidação da formação de estudantes dos cursos de computação. Como principal benefício proporcionado pelo programa de treinamento em desenvolvimento de softwares destaca-se o desenvolvimento em curto prazo de jogos digitais educativos com padrão de mercado e geração de experiência formativa do aluno, que inserido em um contexto que simula o ambiente profissional, irá também desenvolver relações interpessoais e valores profissionais.

Pode-se citar como resultado positivo o desenvolvimento de jogos educativos com intenções pedagógicas claras que podem ser utilizados pela escola. É possível considerar esse trabalho uma experiência piloto válida e que os resultados alcançados servem como subsidio para a realização de novos experimentos.

Como futuros trabalhos temos: aprimorar o programa levando-se em 
consideração os critérios avaliados nessa experiência, de forma que se consiga superar as dificuldades que foram aqui observadas. Além disso, pretendemos melhorar o processo de desenvolvimento de jogos, validando-o através de outras experiências.

\section{Referências}

Begosso, L. R., Begosso, L. C., Poletto, A., da Cunha, D. S., and de Lima, F. C. (2011) "Programa de residência em software". In XIX Workshop de Educação em Informática, Natal, Brasil.

Fabricio J. Barth, Leo Burd, Mauricio Pimentel (2011) "Escritório de projetos: simulando o ambiente de projetos de software em cursos de tecnologia". Faculdade de Tecnologia Bandeirantes - BandTec, Sao Paulo, SP, Brasil.

Gros, Begona (2008) "The impact of digital games in education” First Monday, v.8.

Lara, I. C. M (2014) “Jogando com a Matemática de $5^{\text {a a }} 8^{a}$ série”. São Paulo: Rêspel.

Nascimento, M., Mendonça, A., Guerrero, D., and de Figueiredo, J. (2010). Teaching programming for high school students: A distance education experience. In Frontiers in Education Conference (FIE), 2010 IEEE, pages F1J-1-F1J-6.

Paes, R., Malaquias, R., Guimaraes, M., and Almeira, H. (2013). "Ferramenta para a avaliação de aprendizado de alunos em programação de computadores". In Anais dos Workshops do CBIE 2013, Dourados, MS.

Prensky,M.(2012). “Aprendizagem Baseada em Jogos Digitais”. São Paulo. Editora Senac, $1^{\mathrm{a}}$ edição.

Sá, E.J.V, Teixeira, J.S.F, and Fernandes, C.T (2007) "Design de atividades de aprendizagem que usam Jogos como princípio para Cooperação". In: Anais do XVIII Simpósio Brasileiro de Informática na Educação (SBIE), São Paulo - SP, Brasil.

Sampaio, A. et. al. (2005) "Software Test Program: a Software Residency Experience" In International Conference on Software Engineering. Proceedings of 27th ICSE.

Savi, Rafael and Ulbricht, Vania (2008) "Jogos digitais educacionais: benefícios e desafios". CINTED - UFRGS.

Sutherland, Jeff. (2014) "Scrum - A Arte de Faze o Dobro de Trabalho na Metade do Tempo". $1^{a}$ ed. Leya Brasil, São Paulo.

Tarouco, L. M. R, Roland, L. C, Fabre, M. C. J. M, and Konrath, M. L. P. (2004) "Jogos educacionais". In: Novas Tecnologias na Educação - RENOTE, v.2, n.1.

Torezani, C., Chagas, L., and Tavares, O. (2013). "Newprog - um ambiente online para crianças aprenderem programação de computadores. In XXV Workshop de Informática na Escola (WIE 13), Campinas, SP.

Vivacqua, F. R. (2009) "Fábricas de Software e a Academia: Análise da Formação Acadêmica em Informática no Município do Rio de Janeiro". Dissertação de Mestrado da FGV - Escola Brasileira de Administração Pública e de Empresas. Rio de Janeiro. Disponível em http://virtualbib.fgv.br/dspace/handle/10438/3703

Way, T. P. (2005) “A Company-based Framework for a Software Engineering Course". In Technical Symposium on Computer Science Education. Proceedings of the 36th SIGCSE Technical Symposium on Computer Science Education. St. Louis, USA. 